\title{
Fiscalização de verduras comercializadas no município de Ribeirão Preto, SP
}

\author{
Monitoring of vegetables commercially sold in Ribeirão Preto, SP, Brazil
}

\author{
Osvaldo M. Takayanagui', Carlos D. Oliveira², Alzira M.M. Bergamini ${ }^{3}$, Divani M. Capuano ${ }^{3}$, Madalena H.T. \\ Okino $^{3}$, Luiza H.P. Febrônio², Ana A.M.C. Castro e Silva ${ }^{4}$, Maria A. Oliveira ${ }^{3}$, \\ Eliana G.A. Ribeiro ${ }^{3}$ e Angela M.M. Takayanagui ${ }^{5}$
}

\begin{abstract}
Resumo 0 consumo de verduras cruas constitui importante meio de transmissão de várias doenças infecciosas. Este estudo tem como objetivo a avaliação microbiológica e parasitológica de verduras comercializadas no município de Ribeirão Preto, SP, abrangendo todos os pontos de venda ao consumidor. Do total de 172 estabelecimentos fixos ou ambulantes analisados, 115 (67\%) apresentaram hortaliças com irregularidades: elevada concentração de coliformes fecais em 63\%, presença de Salmonella em $9 \%$ e de enteroparasitas em 33\%. Os pontos de venda com maior freqüência de hortaliças com resultados inadequados foram: mercearias (92\%), CEAGESP (75\%), quitandas (71\%), vendedores ambulantes (71\%), feiras-livres (69\%), supermercados (52\%) e hortas (18\%). O tipo de contaminação apresentou distribuição uniforme em relação aos locais de venda e à variedade da hortaliça. A maioria (61\%) das verduras contaminadas era procedente de hortas localizadas no município de Ribeirão Preto. Considerando a elevada freqüência de contaminação fecal e o potencial risco de doenças veiculadas pelas hortaliças, sugerimos uma vigilância sanitária mais atuante na fiscalização de alimentos oferecidos à população.
\end{abstract}

Palavras-chaves: Verduras. Coliformes fecais. Salmonella. Cisticercose.

\begin{abstract}
The ingestion of raw vegetables represents an important means of transmission of several infectious diseases. The objective of the present study was to perform a microbiological and parasitological evaluation of the vegetables commercially sold in the municipality of Ribeirão Preto, SP, Brazil. Of a total of 172 commercial concerns analyzed, 115 (67\%) presented irregularities in the vegetables they sold, such as elevated concentration of fecal coliforms in 63\%, presence of Salmonella in 9\%, and presence of enteroparasites in $33 \%$. The commercial concerns with the highest frequencies of vegetables showing inadequate results were: grocery stores (92\%), CEAGESP (75\%), fruit and vegetables stores (71\%), traveling vendors (71\%), fairs (69\%), supermarkets (52\%), and vegetable gardens (18\%). The type of contamination was uniformly distributed among these commercial concerns. Most of the contaminated vegetables (61\%) were from gardens located in the municipality of Ribeirão Preto. Considering the high frequency of fecal contamination and the potential risk of disease transmitted by vegetables, we suggest greater enforcement in the sanitary surveillance of the food offered to the population.
\end{abstract}

Key-words: Lettuce. Vegetable. Fecal coliforms. Salmonella. Cysticercosis.

O consumo de verduras cruas constitui importante meio de transmissão de várias doenças infecciosas e parasitárias pela freqüente prática de irrigação de hortas com água contaminada por matéria fecal ou mesmo adubadas com dejetos humanos ${ }^{6262728}$. Este estudo tem como objetivo a avaliação microbiológica e parasitológica de verduras comercializadas no município de Ribeirão Preto, SP, abrangendo todos os pontos de venda ao consumidor.

\footnotetext{
1. Departamento de Neurologia da Faculdade de Medicina de Ribeirão Preto da Universidade de São Paulo, Ribeirão Preto, SP; 2. Divisão de Vigilância Sanitária, Secretaria da Saúde do Município de Ribeirão Preto; 3. Instituto Adolfo Lutz, Laboratório I de Ribeirão Preto, Secretaria da Saúde do Estado de São Paulo; 4. Divisão de Vigilância Epidemiológica, Secretaria da Saúde do Município de Ribeirão Preto; 5 . Disciplina de Saúde Pública, Escola de Enfermagem de Ribeirão Preto, Universidade de São Paulo, Ribeirão Preto, SP

Endereço para correspondência: Dr. Osvaldo M. Takayanagui. Deptº de Neurologia/FMRP/USP, 14048-900 Ribeirão Preto, SP

Tel: 5516 3623-3996; fax: 5516 3633-0866

e-mail: otakay@rnp.fmrp.usp.br

Recebido para publicação em 17/12/99.
} 


\section{MATERIAL E MÉTODOS}

No período de julho de 1997 a julho de 1998, todos os pontos de venda de hortaliças: supermercados, feiraslivres, quitandas, CEAGESP (Companhia de Entrepostos e Armazéns Gerais do Estado de São Paulo), hortas e vendedores ambulantes foram avaliados por fiscais da Divisão de Vigilância Sanitária da Secretaria Municipal da Saúde, com coleta aleatória de 2 pés de verduras de folha, preferencialmente a alface (Lactuca sativa) ou, na sua ausência, almeirão (Chicorium intybus), rúcula (Eruca sativa), agrião em (Nasturtium officinale) ou chicória (Chicorium endivia). A verdura foi acondicionada individualmente em sacos plásticos de primeiro uso, sem contato manual, e encaminhada ao Instituto Adolfo Lutz de Ribeirão Preto para análises microbiológica e parasitológica, segundo Gelli et al ${ }^{11}$ e Marzochi ${ }^{23}$, com algumas modificações.

A análise microbiológica foi fundamentada na determinação do número mais provável de bactéria do grupo coliforme de origem fecal (NMP/g) e na pesquisa de Salmonella sp, segundo metodologia preconizada pela American Public Health Association ${ }^{2}$ e de acordo com a legislação brasileira em vigor. A Secretaria Nacional de Vigilância Sanitária estabelece, para hortaliças frescas, refrigeradas ou congeladas, um limite de tolerância do NMP de coliformes fecais em até $200 / g$ e a ausência de Salmonella em $25 \mathrm{~g}$ do produto $^{5}$. O cálculo do NMP de coliformes fecais foi efetuado com o auxílio da tabela de Hoskins ${ }^{14}$.

A análise parasitológica foi realizada após duas lavagens. Na primeira, por enxaguadura, o saco plástico contendo a verdura foi agitado manualmente por 30 segundos após introdução de $250 \mathrm{ml}$ de água destilada. Na segunda, após desfolhamento, cada folha da verdura foi esfregada com um pincel chato $n^{\circ} 16$ num recipiente de vidro com $250 \mathrm{ml}$ de água destilada. A água das lavagens foi deixada em repouso em cálice cônico por 24 horas após filtragem em gaze de 8 dobras. O sedimento então obtido foi analisado ao microscópio por exame direto e após centrífugo-flutuação em sulfato de zinco ${ }^{30}$. Outra parte do sedimento foi utilizada na pesquisa de oocistos de Cryptosporidium sp por adição de formalina tamponada a $10 \%$, após concentração pela técnica do formol-éter ${ }^{8}$ e coloração do esfregaço por Kinyoun ${ }^{17}$.

A análise estatística foi baseada no teste de comparação de proporções múltiplas ${ }^{10}$, adotando $\mathrm{p} \leq 0,05$ como nível de significância.

\section{RESULTADOS}

Do total de 172 pontos de venda analisados, 115 (67\%) apresentaram hortaliças com irregularidades, expostas na Tabela 1.

O exame microbiológico revelou elevada concentração de coliformes fecais, acima do máximo permitido pela legislação, em 108 (63\%) amostras e presença de Salmonella em 15 (9\%). O NMP de coliformes fecais chegou a mais de 10.800/g numa amostra de alface comercializada em feira-livre. Os sorotipos de Salmonella foram: S. Javiana em 5 amostras, S. Oranienburg em 2, S. Anatum em 1, $S$. Emek, lisina negativa em 1, S. Infantis em 1, S. Morehead em 1, S. Panamá em 1, S. Typhimurium em 1, S. entérica subespécie diarizonae 6:i:z em 1 e $S$. Agona em outra.
As análises parasitológicas evidenciaram presença de vários enteroparasitas em 57 (33\%) amostras: Entamoeba sp em 44, ancilostomídeos em 27, Ascaris spem 3, Giardia sp em 4, Cryptosporidium sp em 2, Hymenolepis nana em 2 e Toxocara sp em outra. Uma das amostras revelou a presença da forma trofozoíta de Giardia sp.

O percentual de irregularidades nas hortas foi significativamente inferior aos demais pontos de venda. O tipo de contaminação, isto é, coliformes fecais, Salmonella e enteroparasitas, apresentou distribuição uniforme em relação ao tipo de estabelecimento comercial e à variedade de verdura (Tabela 2), não havendo diferenças estatisticamente significativas.

Tabela 1 - Irregularidades encontradas nos exames microbiológico e parasitológico de verduras e os diferentes tipos de pontos de venda.

\begin{tabular}{|c|c|c|c|c|c|c|c|c|c|}
\hline \multirow[t]{2}{*}{ Irregularidades } & \multirow{2}{*}{$\begin{array}{c}\text { Quitanda } \\
\mathrm{n}=63\end{array}$} & \multirow{2}{*}{$\begin{array}{c}\text { Supermercado } \\
n=38\end{array}$} & \multirow{2}{*}{$\begin{array}{c}\text { Mercearia } \\
n=25\end{array}$} & \multirow{2}{*}{$\begin{array}{c}\text { Feira-livre } \\
n=16\end{array}$} & \multirow{2}{*}{$\begin{array}{c}\text { CEAGESP } \\
n=12\end{array}$} & \multirow{2}{*}{$\begin{array}{c}\text { Ambulante } \\
\mathrm{n}=7\end{array}$} & \multirow{2}{*}{$\begin{array}{l}\text { Horta } \\
\mathrm{n}=11\end{array}$} & \multicolumn{2}{|c|}{ Total } \\
\hline & & & & & & & & $\mathrm{n}=172$ & $\%$ \\
\hline CF & 24 & 10 & 9 & 3 & 3 & 1 & 1 & 51 & 30 \\
\hline Salmonella & 1 & - & 1 & - & - & - & - & 2 & 1 \\
\hline $\mathrm{CF}+$ Salmonella & - & 1 & 2 & 1 & - & 1 & - & 5 & 3 \\
\hline Parasita & 1 & 1 & - & - & - & - & - & 2 & 1 \\
\hline $\mathrm{CF}+$ parasita & 15 & 7 & 9 & 6 & 6 & 3 & 1 & 47 & 27 \\
\hline Salmonella + parasita & 1 & - & 1 & 1 & - & - & - & 3 & 2 \\
\hline$\underline{\mathrm{CF}}+$ Salmonella + parasita & 3 & 1 & 1 & - & - & - & - & 5 & 3 \\
\hline Total & $45(71 \%)$ & 20 (53\%) & 23 (92\%) & $11(69 \%)$ & $9(75 \%)$ & $5(71 \%)$ & $2(18 \%)$ & 115 & $(67 \%)$ \\
\hline
\end{tabular}

CEAGESP = Companhia de Entrepostos e Armazéns Gerais do Estado de São Paulo; CF= coliformes fecais. 
Tabela 2 - Tipo de contaminação: microbiológica e/ou parasitológica de acordo com a variedade de verduras.

\begin{tabular}{|c|c|c|c|c|c|}
\hline \multirow[t]{2}{*}{ Verdura } & \multirow{2}{*}{$\begin{array}{c}\text { № de verduras } \\
\text { analisadas }\end{array}$} & \multirow{2}{*}{$\begin{array}{l}\text { № de verduras } \\
\text { contaminadas }\end{array}$} & \multicolumn{3}{|c|}{ Contaminação } \\
\hline & & & microbiológica ( $\mathrm{n}^{\circ}$ ) & parasitológica $\left(\mathrm{n}^{\circ}\right.$ ) & ambas $\left(n^{\circ}\right)$ \\
\hline alface & 139 & 98 & 52 & 2 & 44 \\
\hline almeirão & 19 & 10 & 3 & - & 7 \\
\hline rúcula & 10 & 6 & 2 & - & 4 \\
\hline chicória & 2 & 1 & 1 & - & - \\
\hline agrião & 2 & - & - & - & - \\
\hline Total & 172 & 115 & 58 & 2 & 55 \\
\hline
\end{tabular}

A procedência das verduras contaminadas foi de produtores de Ribeirão Preto em $61 \%$ e de outras localidades em $39 \%$. Os resultados irregulares de hortas localizadas em outros municípios foram encaminhados às respectivas Secretarias de Saúde para providências cabíveis.

\section{DISCUSSÃO}

As doenças veiculadas por alimentos são resultantes predominantemente do ciclo de contaminação fecal/oral e seu controle tem recebido atenção cada vez maior em todo o mundo ${ }^{34151625}$. No Brasil, não obstante a relevância e atualidade do problema, são poucos os trabalhos avaliando a qualidade das hortaliças consumidas pela população.

Nosso estudo evidenciou que $67 \%$ dos pontos de venda comercializam hortaliças contaminadas com concentração de coliformes fecais acima do máximo permitido pela legislação em $63 \%$, presença de Salmonella em $9 \%$ e de enteroparasitas em 33\%. A detecção de Entamoeba sp e de ancilostomídeos, nem sempre patogênicos ao homem, foi valorizada somente em concomitância à elevada concentração de coliformes fecais.

O percentual de irregularidades observado em nosso material não diferiu substancialmente do descrito na literatura nacional. Gelli et al ${ }^{11}$, analisando hortaliças comercializadas no município de São Paulo, constataram presença de Escherichia coliem 74,4\%, de ancilostomídeos em $59,2 \%$ e de Strongyloides sp em $59,2 \%$ das amostras, com ausência de Salmonella. Oliveira e Germano ${ }^{26}$ evidenciaram, também em São Paulo, vários tipos de helmintos em $32 \%$ das amostras de alface lisa e em $66 \%$ de agrião e protozoários em $18 \%$ e $60 \%$, respectivamente. Por sua vez, Silva et $\mathrm{a}^{32}$ evidenciaram, em supermercados do Rio de Janeiro, contaminação de verduras com ovos de helmintos em $21,4 \%$ das amostras e Guilherme et $\mathrm{a}^{12}$, em Maringá PR, em 16,6\%. Estes dados contrastam com a baixa $(6,2 \%)$ freqüência observada por Mesquita et al ${ }^{24}$, em Niterói e no Rio de Janeiro.

A contaminação de hortaliças pode ocorrer na horta, resultante da utilização de água de irrigação ou adubos inadequados, no transporte ou por manipulação nos pontos de venda e as sucessivas manipulações aumentam as chances de contaminação. A freqüência significativamente mais baixa de contaminação nas hortas em relação aos demais pontos de venda pode ser justificada por constituir o ponto inicial da cadeia de comércio e, principalmente, pelo programa de fiscalização de hortas implantado no município há 3 anos $^{34}$. A presença da forma trofozoíta de Giardia sp sugere que a contaminação tenha sido evento recente, provavelmente por manipulação ${ }^{29}$ no ponto de venda, visto ser esta forma muito frágil, não sobrevivendo por mais de 15 a 20 minutos no meio externo ${ }^{7}$.

Constatamos vários sorotipos de Salmonella cuja importância não se restringe a quadros de gastrenterite mas também, como potenciais agentes causais de grave bacteremia ${ }^{31}$ e de meningite resistente a múltiplos antibióticos ${ }^{9}$.

Ressaltamos a constatação de oocistos de Cryptosporidium sp, um protozoário emergente que passou a receber maior atenção com o advento da aids (síndrome da imunodeficiência adquirida) por ser responsável por infecção oportunista causador de quadros diarreicos graves em indivíduos imunocomprometidos ${ }^{131821}$, particularmente em crianças ${ }^{122}$ e por surtos de diarréia por contaminação de água de abastecimento público ${ }^{19}$.

A detecção de ovos de Toxocara spindica contaminação de verduras com fezes de canídeos ou felídeos. Sua importância ao homem reside no fato de sua forma larvária poder acarretar manifestações da síndrome da larva migrans visceralis, incluindo graves complicações pulmonares, oculares e neurológicas ${ }^{20}$.

A despeito da não constatação de ovos de Taenia sp, descrita por Marzochi ${ }^{23}$, Silva et al ${ }^{32}$ e Oliveira e Germano ${ }^{26}$, a elevada freqüência de contaminação fecal indica o potencial risco de transmissão de ovos da Taenia solium através do consumo de verduras cruas, podendo justificar a alta prevalência da cisticercose no nosso município ${ }^{33}$.

A legislação vigente sobre o comércio de hortaliças constitui fator limitante para uma atuação fiscalizadora mais rigorosa da Vigilância Sanitária pois a evidência de contaminação, por si, não configura situação ilícita do ponto de vista legal. Somente os proprietários das hortas estão sujeitos a penalidades que incluem autos de infração, multas pecuniárias e até mesmo interdição, motivos maiores do êxito do programa de fiscalização 
de hortas no nosso município ${ }^{34}$. Em contraposição, nos demais pontos de venda, a Vigilância Sanitária não está respaldada para qualquer ação mais restritiva, limitandose a orientações sobre educação sanitária.

A maioria (61\%) das verduras contaminadas era proveniente de hortas localizadas no município de Ribeirão Preto, sendo as demais (39\%) de outras localidades. Aquelas localizadas no município foram fiscalizadas pela Vigilância Sanitária para análise de novas amostras de verduras assim como da água de irrigação (não mostrada no presente estudo). Os resultados inadequados de verduras procedentes de outros municípios foram enviados às respectivas Secretarias de Saúde com sugestão de vistoria das hortas.
Considerando a elevada freqüência de contaminação fecal e o potencial risco de doenças veiculadas pelos alimentos, sugerimos o fortalecimento do sistema de vigilância sanitária para fiscalização de alimentos oferecidos à população, incluindo legislação adequada ${ }^{15}$ e a obrigatoriedade do exame coproparasitológico na emissão e renovação da carteira de saúde dos manipuladores de alimentos. São também relevantes as ações educativas sobre os preceitos básicos de higiene pessoal aos produtore ${ }^{12}$ e manipuladores de alimentos e, para a população em geral, orientação sobre a importância da lavagem cuidadosa e desinfecção das hortaliças antes do consumo.

\section{AGRADECIMENTOS}

Aos Srs. Luiz Antonio Mafud, Antonio José Beordo e Vanderlei Greggi, fiscais sanitários da Divisão de Vigilância Sanitária da Secretaria da Saúde de Ribeirão Preto, pelo incansável trabalho em campo; às Sras. Maria José C.B. Bettini, Solange A.V. Oliveira e Maria Clarice Errera do Instituto Adolfo Lutz, Laboratório I de Ribeirão Preto e às Dras. Ana Célia Mangini, Sueli A. Fernandes e Ana T. Tavechio do Instituto Adolfo Lutz, Laboratório Central de São Paulo, pelo apoio na análise laboratorial.

\section{REFERÊNCIAS BIBLIOGRÁFICAS}

1. Agnew DG, Lima AA, Newman RD, Wuhib T, Moore RD, Guerrant $\mathrm{RL}$, Sears CL. Cryptosporidiosis in northeastern Brazilian children: association with increased diarrhea morbidity. Journal of Infectious Diseases 177:754-760, 1998.

2. American Public Health Association. Technical Commitee on Microbiological Methods for Foods. In: Vanderzant C, Splittstoesser DF (eds) Compendium of methods for the microbiological examination of foods. $3^{\text {rd }}$ edition, American Public Health Association, Washington, p. 336-383, 1992.

3. Blaser MJ. How safe is our food? The New England Journal of Medicine 334:1324-1326, 1996.

4. Borgdorff MW, Motarjemi Y. Surveillance of foodborne diseases: what are the options? World Health Statistics Quarterly 50:1223, 1997.

5. Divisão Nacional de Vigilância Sanitária de Alimentos da Secretaria Nacional de Vigilância Sanitária. Diário Oficial da União, Portaria no 451 de 19 de Setembro de 1997, Seção I, p. 4-13, Brasília, 1998.

6. Centers for Disease Control and Prevention. Incidence of foodborne illnesses. Morbidity and Mortality Weekly Report 47:782-786, 1998.

7. Cimerman B, Cimerman S. Giardíase. In: Veronesi R, Focaccia R (eds) Tratado de Infectologia, Atheneu, São Paulo, p. 12141216, 1996.

8. Dias RMTS, Mangini ACS, Torres DMAGV, Corrêa MOA, Lupetti N, Corrêa FMA, Chieffi PP. Cryptosporidiosis among patients with acquired immunodeficiency syndrome (AIDS) in the county of São Paulo, Brazil. Revista do Instituto de Medicina Tropical de São Paulo 30:310-312, 1998.

9. Esper MRNR, Freitas AM, Fernandes AS, Neme SN, Tavechio AT, Romão MM, Caféml. Salmonella: sorotipos identificados das cepas isoladas de pacientes hospitalizados e não hospitalizados, na região de Presidente Prudente, SP, no período de 1978-1997. Revista do Instituto Adolfo Lutz 57:45-50, 1998.
10. Fleiss JL. Statistical methods for rates and proportions. $2^{\text {nd }}$ edition. John Wiley \& Sons, New York, 1981.

11. Gelli DS, Tachibana T, Oliveira IR, Zamboni CQ, Pacheco JA, Spiteri N. Condições higiênico-sanitárias de hortaliças comercializadas na cidade de São Paulo, SP, Brasil. Revista do Instituto Adolfo Lutz 39:37-43, 1979.

12. Guilherme AL, Araújo SM, Falavigna DL, Pupulim AR, Dias ML, Oliveira HS, Maroco E, Fukushigue Y. Prevalência de enteroparasitas em horticultores e hortaliças da Feira do Produtor de Maringá, Paraná. Revista da Sociedade Brasileira de Medicina Tropical 32:405-411, 1999.

13. Guizelini E, Amato Neto V. Pesquisa de oocistos de Cryptosporidium sp nas fezes diarréicas de aidéticos e de crianças e adultos imunocompetentes, em São Paulo. Revista do Hospital das Clínicas da Faculdade de Medicina da Universidade de São Paulo 47:150-152, 1992.

14. International Commission on Microbiological Specifications for Foods. Microorganisms in foods: their significance and methods of enumeration. University of Toronto, Toronto, 1978.

15. Käferstein F, Abdussalam M. Food safety in the $21^{\text {st }}$ century. Bulletin of the World Health Organization 77:347-351, 1999.

16. Käferstein FK. Food safety: a commonly underestimated public health issue. World Health Statistics Quarterly 50:3-4, 1997.

17. Lennette EH, Balows A, Hausler WJ, Truant JP. Manual of clinical microbiology. $4^{\text {th }}$ edition, American Society for Microbiology, Washington, 1985.

18. Lumadue JA, Manabe YC, Moore RD, Belitsos PC, Sears CL, Clark DP. A clinicopathologic analysis of AIDS-related cryptosporidiosis. AIDS 12:2459-2466, 1998.

19. MacKenzie WR, Schell WL, Blair KA, Addiss DG, Peterson DE, Hoxie NJ, Kazmierczak JJ, Davis JP. Massive outbreak of waterborne Cryptosporidium infection in Milwaukee, Wisconsin: recurrence of illness and risk of secondary transmission. Clinical Infectious Diseases 21:57-62, 1995. 
20. Magnaval JF, Galindo V, Glickman LT, Clanet M. Human Toxocara infection of the central nervous system and neurological disorders: a case-control study. Parasitology 115:537-543, 1997.

21. Manabe YC, Clark DP, Moore RD, Lumadue JA, Dahlman HR, Belitsos PC, Chaisson RE, Sears CL Cryptosporidiosis in patients with AIDS: correlates of disease and survival. Clinical Infectious Diseases 27:536-542, 1998.

22. Mangini AC, Dias RM, Grisi SJ, Escobar AM, Torres DM, Zuba IP, Quadros CM, Chieffi PP. Parasitismo por Cryptosporidium sp em crianças com diarréia aguda. Revista do Instituto de Medicina Tropical de São Paulo 34:341-345, 1992.

23. Marzochi MCA. Estudo epidemiológico da poluição por enteroparasitas em áreas de horticultura da cidade de Ribeirão Preto, SP, Brasil. Tese de Doutorado, Universidade Estadual de Londrina, Londrina, PR, 1974.

24. Mesquita VC, Serra CM, Bastos OM, Uchôa CM. Contaminação por enteroparasitas em hortaliças comercializadas nas cidades de Niterói e Rio de Janeiro, Brasil. Revista da Sociedade Brasileira de Medicina Tropical 32:363-366, 1999.

25. Motarjemi Y, Käferstein FK. Global estimation of foodborne diseases. World Health Statistics Quarterly 50:5-11, 1997.

26. Oliveira CA, Germano PM. Estudo da ocorrência de enteroparasitas em hortaliças comercializadas na região metropolitana de São Paulo, SP, Brasil. I- Pesquisa de helmintos. Revista de Saúde Pública 26:283-289, 1992.

27. Oliveira CAF, Germano PML. Estudo da ocorrência de enteroparasitas em hortaliças comercializadas na região metropolitana de São Paulo, SP, Brasil. II- Pesquisa de protozoários intestinais. Revista de Saúde Pública 26:332-335, 1992.

28. Pattoli D, Paim GV. Enteroparasitas de águas de irrigação de hortas que abastecem o município de São Paulo. Revista Paulista de Medicina 68:241, 1966.

29. Petrsen I, Cartter RML, Hadler LJ. A food-borne outbreak of Giardia lamblia. Journal of Infectious Diseases 157:846-848, 1988.

30. Rey L. Parasitologia. $2^{a}$ edição, Guanabara Koogan, Rio de Janeiro, 1991

31. Shimoni Z, Pitlik S, Leibovici L, Samra Z, Konigsberger H, Drucker M, Agmon V, Ashkenazi S, Weinberger M. Nontyphoid Salmonella bacteremia: age-related differences in clinical presentation, bacteriology, and outcome. Clinical Infectious Diseases 28:822827, 1999.

32. Silva JP, Marzochi MC, Camillo-Coura L, Messias AA, Marques $S$. Estudo da contaminação por enteroparasitas em hortaliças comercializadas nos supermercados da cidade do Rio de Janeiro. Revista da Sociedade Brasileira de Medicina Tropical 28:237241, 1995.

33. Takayanagui OM, Castro e Silva AA, Santiago RC, Odashima NS, Terra VC, Takayanagui AM. Notificação compulsória da cisticercose em Ribeirão Preto-SP. Arquivos de Neuropsiquiatria 54:557-564, 1996.

34. Takayanagui OM, Febrônio LH, Bergamini AM, Okino MH, Castro e Silva AA, Santiago R, Capuano DM, Oliveira MA, Takayanagui AM. Fiscalização de hortas produtoras de verduras no município de Ribeirão Preto, SP. Revista da Sociedade Brasileira de Medicina Tropical 33:169-174, 2000. 